\title{
Article \\ Weighted Estimates for Iterated Commutators of Riesz Potential on Homogeneous Groups
}

\author{
Daimei Chen, Yanping Chen * and Teng Wang
}

check for updates

Citation: Chen, D.; Chen, Y.; Wang, T. Weighted Estimates for Iterated Commutators of Riesz Potential on Homogeneous Groups. Mathematics 2021, 9, 2421. https://doi.org/ $10.3390 /$ math 9192421

Academic Editor: Christopher Goodrich

Received: 24 August 2021 Accepted: 23 September 2021 Published: 29 September 2021

Publisher's Note: MDPI stays neutral with regard to jurisdictional claims in published maps and institutional affiliations.

Copyright: (c) 2021 by the authors. Licensee MDPI, Basel, Switzerland. This article is an open access article distributed under the terms and conditions of the Creative Commons Attribution (CC BY) license (https:// creativecommons.org/licenses/by/ $4.0 /)$.
Department of Applied Mathematics, School of Mathematics and Physics, University of Science and Technology Beijing, Beijing 100083, China; daimeich@126.com (D.C.); tengwustb@126.com (T.W.)

* Correspondence: yanpingch@ustb.edu.cn

Abstract: In this paper, we study the two weight commutators theorem of Riesz potential on an arbitrary homogeneous group $\mathbb{H}$ of dimension $N$. Moreover, in accordance with the results in the Euclidean space, we acquire the quantitative weighted bound on homogeneous group.

Keywords: commutators; Riesz potential; homogeneous group

\section{Introduction and Main Results}

Suppose $\mathbb{H}$ is a nilpotent Lie group, which has the multiplication, inverse, expansion and norm configurations $(x, y) \mapsto x y, x \mapsto x^{-1},(t, x) \mapsto t \circ x, x \mapsto \rho(x)$ for $x, y \in \mathbb{H}, t>0$, respectively, then we call $\mathbb{H}$ being a homogeneous group (see [1] or [2]). The multiplication and inverse operations are polynomials and $t$-action is an automorphism of the group structure, where $t$ is of the form

$$
t \circ\left(x_{1}, \ldots, x_{n}\right)=\left(t^{\beta_{1}} x_{1}, \ldots, t^{\beta_{n}} x_{n}\right)
$$

for some constants $0<\beta_{1} \leq \beta_{2} \leq \ldots \leq \beta_{n}$. Besides, $\rho(x):=\max _{1 \leq j \leq n}\left\{\left|x_{j}\right|^{1 / \beta_{j}}\right\}$ is a norm linked to the expansion configuration. We call the value $N=\sum_{j=1}^{n} \beta_{j}$ the dimensionality of $\mathbb{H}$. In addition to the Euclidean structure, $\mathbb{H}$ is equipped with a homogeneous nilpotent Lie group structure, where Lebesgue measure is a bi-invariant Haar measure, the identity is the origin $0, x^{-1}=-x$ and multiplication $x y, x, y \in \mathbb{H}$, satisfies

(1) $(a x)(b x)=a x+b x, x \in \mathbb{H}, a, b \in \mathbb{R}$;

(2) $t \circ(x y)=(t \circ x)(t \circ y), x, y \in \mathbb{H}, t>0$;

(3) if $z=x y$, then $z_{k}=P_{k}(x, y)$, where $P_{1}(x, y)=x_{1}+y_{1}$ and $P_{k}(x, y)=x_{k}+y_{k}+$ $P_{k}(x, y)$ for $k \geq 2$ with a polynomial $P_{k}(x, y)$ depending only on $x_{1}, \cdots, x_{k-1}, y_{1}, \cdots, y_{k-1}$.

Finally, the Heisenberg group on $\mathbb{R}^{3}$ is an example of a homogeneous group. If we define the multiplication

$$
(x, y, u)\left(x^{\prime}, y^{\prime}, u^{\prime}\right)=\left(x+x^{\prime}, y+y^{\prime}, u+u^{\prime}+\left(x y^{\prime}-y x^{\prime}\right) / 2\right),
$$

$(x, y, u)\left(x^{\prime}, y^{\prime}, u^{\prime}\right) \in \mathbb{R}^{3}$, the $\mathbb{R}^{3}$ with this group law is the Heisenberg group $\mathbb{H}_{1}$; a dilation is defined by $t \circ(x, y, u)=\left(t x, t y, t^{2} u\right)$, that is the parameters $\beta_{1}=1, \beta_{2}=1, \beta_{3}=2$.

Definition 1. Let $w(x)$ is a function on $\mathbb{H}$, which is non-negative locally integrable. For $1<p<$ $\infty$, we call that $w$ is an $A_{p}$ weight, denoted by $w \in A_{p}$, if

$$
[w]_{A_{p}}:=\sup _{B}\left(\frac{1}{|B|} \int_{B} w(x) d x\right)\left(\frac{1}{|B|} \int_{B}\left(\frac{1}{w}\right)^{\frac{1}{p-1}} d x\right)^{p-1}<\infty,
$$

The supremum here is taken over of all balls $B \subset \mathbb{H}$. We call that the quantity $[w]_{A_{p}}$ is the $A_{p}$ constant of $w$. For $p=1$, if $M(w)(x) \leq c w(x)$ for a.e. $x \in \mathbb{H}$, then we say that $w$ is an $A_{1}$ weight, 
denoted by $w \in A_{1}$, where $M$ represents the Hardy-Littlewood maximal function. In addition, let $A_{\infty}:=\cup_{1 \leq p \leq \infty} A_{p}$, then we have

$$
[w]_{A_{\infty}}:=\sup _{B}\left(\frac{1}{|B|} \int_{B} w d x\right) \exp \left(\frac{1}{|B|} \int_{B} \log \left(\frac{1}{w}\right) d x\right)<\infty .
$$

Definition 2. Let $x \in \mathbb{H}$, and $w(x)$ be a non-negative locally integrable function. For $1<p<$ $q<\infty, w \in A_{p, q}$ if

$$
[w]_{A_{p, q}}:=\sup _{B}\left(\frac{1}{|B|} \int_{B} w^{q}\right)\left(\frac{1}{|B|} \int_{B} w^{-p^{\prime}}\right)^{\frac{q}{p^{\prime}}}<\infty
$$

where $p^{\prime}$ is the conjugate exponent of $p$, that is $\frac{1}{p}+\frac{1}{p^{\prime}}=1$.

Definition 3. Suppose $w \in A_{\infty}$. Let $b \in L_{l o c}^{1}(\mathbb{H})$, then $b(x) \in B M O_{w}(\mathbb{H})$ if

$$
\|b\|_{B M O_{w}(\mathbb{H})}:=\sup _{B} \frac{1}{w(B)} \int_{B}\left|b(x)-b_{B}\right| d x<\infty,
$$

where $b_{B}:=\frac{1}{|B|} \int_{B} b(x) d x$ and the supremum is taken over of all balls $B \subset \mathbb{H}$.

We now review the definition of Riesz potential on homogeneous group. For $0<\alpha<N$,

$$
I_{\alpha} f(x):=\int_{\mathbb{H}} \frac{f(y)}{\rho\left(x y^{-1}\right)^{N-\alpha}} d y,
$$

and the corresponding associated maximal function $M_{\alpha}$ by

$$
M_{\alpha} f(x)=\sup _{x \in B} \frac{1}{|B|^{1-\frac{\alpha}{N}}} \int_{B}|f(y)| d y .
$$

The reason why we study the weighted estimates for these operators is because they have a wide range of applications in partial differential equations, Sobolev embeddings or quantum mechanics (see [3] or [4]).

Muckenhoupt and Wheeden [5] are the first scholars to study the Riesz potential. When $\mathbb{H}$ is an isotropic Euclidean space, Muckenhoupt and Wheeden [5] show that $I_{\alpha}$ is bounded from $L^{p}\left(w^{p}\right)$ to $L^{q}\left(w^{q}\right)$ for $1<p<\frac{n}{\alpha}, \frac{1}{q}=\frac{1}{p}-\frac{\alpha}{n}, w \in A_{p, q}$. Moreover, the sharp constant in this inequality was given in [6]:

$$
\left\|I_{\alpha}\right\|_{L^{p}\left(w^{p}\right) \rightarrow L^{q}\left(w^{q}\right)} \leq C[w]_{A_{p, q}}^{\left(1-\frac{\alpha}{n}\right) \max \left(1, \frac{p \prime}{q}\right)} .
$$

Definition 4. Suppose $b \in L_{l o c}^{1}(\mathbb{H}), f \in L^{p}(\mathbb{H})$. Let $\left[b, I_{\alpha}\right]$ be the commutator defined by

$$
\left[b, I_{\alpha}\right] f(x):=b(x) I_{\alpha}(f)(x)-I_{\alpha}(b f)(x) .
$$

The iterative commutators $\left(I_{\alpha}\right)_{b}^{m}, m \in \mathbb{N}$, are defined naturally by

$$
\left(I_{\alpha}\right)_{b}^{m} f(x):=\left[b,\left(I_{\alpha}\right)_{b}^{m-1}\right] f(x),\left(I_{\alpha}\right)_{b}^{1} f(x):=\left[b, I_{\alpha}\right] f(x) .
$$

In 2016, Holmes, Rahm and Spencer [7] prove that

$$
\left[b, I_{\alpha}\right]: L_{w^{p}}^{p}\left(\mathbb{R}^{n}\right) \rightarrow L_{\lambda q}^{q}\left(\mathbb{R}^{n}\right) \Leftrightarrow b \in B M O_{\mu}\left(\mathbb{R}^{n}\right),
$$


where $1<p<\frac{n}{\alpha}, \frac{1}{q}=\frac{1}{p}-\frac{\alpha}{n}, w, \lambda \in A_{p, q}, \mu=\frac{w}{\lambda}$. Later, the quantitative estimates for iterated commutators of fractional integrals was obtained by N. Accomazzo, J. C. MartínezPerales and I. P. Rivera-Ríos [8].

In 2013, Sato [9] gave the estimates for singular integrals on homogeneous groups. In [10], X. T. Duong, H. Q. Li and J. Li established the Bloom-type two weight estimates for the commutator of Riesz transform on stratified Lie groups. Moreover, Z. Fan and $\mathrm{J}$. Li [11] obtained the quantitative weighted estimates for rough singular integrals on homogeneous groups.

Motivated by the above estimates, we investigate the quantitative weighted estimation for the higher order commutators of fractional integral operators on homogeneous groups.

In this paper, our main result is the follow theorem.

Theorem 1. Let $0<\alpha<N$ and $1<p<\frac{N}{\alpha}$, q defined by $\frac{1}{q}+\frac{\alpha}{N}=\frac{1}{p}$, and $m$ is a positive integer. Assume that $\mu, \lambda \in A_{p, q}$ and that $v=\frac{\mu}{\lambda}$.

1. If $b \in B M O_{v^{1 / m}}(\mathbb{H})$, then

$$
\left\|\left(I_{\alpha}\right)_{b}^{m} f\right\|_{L_{\lambda}^{q}(\mathbb{H})} \leq C_{m, N, \alpha, p}\|b\|_{B M O_{v^{1 / m}}(\mathbb{H})^{k}}^{\kappa_{m}}\|f\|_{L_{\mu^{p}}^{p}(\mathbb{H})^{\prime}}
$$

where

$$
\kappa_{m}=\sum_{k=0}^{m}\left(\begin{array}{c}
m \\
k
\end{array}\right)\left([\lambda]_{A_{p, q}}^{\frac{k}{m}}[\mu]_{A_{p, q}}^{\frac{m-k}{m}}\right)^{\left(1-\frac{\alpha}{N}\right) \max \left\{1, \frac{p^{\prime \prime}}{q}\right\}} A(m, k) B(m, k)
$$

and

$$
\begin{gathered}
A(m, k) \leq\left(\left[\lambda^{q}\right]_{A_{q}}^{\frac{m+k+1}{2}}\left[\mu^{q}\right]_{A_{q}}^{\frac{m-k-1}{2}}\right)^{\frac{m-k}{m} \max \left\{1, \frac{1}{q-1}\right\}}, \\
B(m, k) \leq\left(\left[\lambda^{p}\right]_{A_{p}}^{\frac{k-1}{2}}\left[\mu^{p}\right]_{A_{p}}^{m-\frac{k-1}{2}}\right)^{\frac{k}{m} \max \left\{1, \frac{1}{p-1}\right\}} .
\end{gathered}
$$

2. For every $b \in L_{\text {loc }}^{1}(\mathbb{H})$, if $\left(I_{\alpha}\right)_{b}^{m}$ is bounded from $L_{\mu^{p}}^{p}(\mathbb{H})$ to $L_{\lambda^{q}}^{q}(\mathbb{H})$, then $b \in B M O_{v^{1 / m}}(\mathbb{H})$ with

$$
\|b\|_{B M O_{v^{1 / m}}(\mathbb{H})}^{m} \lesssim\left\|\left(I_{\alpha}\right)_{b}^{m}\right\|_{L_{\mu^{p}}^{p}(\mathbb{H}) \rightarrow L_{\lambda q}^{q}(\mathbb{H})} \cdot
$$

\section{Domination of the Iterated Commutators by Sparse Operators}

\subsection{A System of Dyadic Cubes}

We define a left-unchanged analogous-distance $d$ on $\mathbb{H}$ by $d(x, y)=\rho\left(x^{-1} y\right)$, which signifies that there has a constant $A_{0} \geq 1$ such that for any $x, y, z \in \mathbb{H}$,

$$
d(x, y) \leq A_{0}[d(x, z)+d(z, y)] .
$$

Next, let $B(x, r):=\{y \in \mathbb{H}: d(x, y)<r\}$ be the open ball which is centered on $x \in \mathbb{H}$ and $r>0$ is the radius.

Let $\mathscr{A}_{k}$ be $k$-th denumerable index set. A denumerable class $\mathcal{D}:=\cup_{k \in \mathbb{Z}} \mathcal{D}_{k}, \mathcal{D}_{k}:=$ $\left\{Q_{\beta}^{k}: \beta \in \mathscr{A}_{k}\right\}$, of Borel sets $Q_{\beta}^{k} \subseteq \mathbb{H}$ is known as a set of dyadic cubes with arguments $\delta \in(0,1)$ and $0<a_{1} \leq A_{1}<\infty$ if it has the characteristics below:

(1) $\mathbb{H}=\cup_{\beta \in \mathscr{A}_{k}} Q_{\beta}^{k}$ (disjoint union) for all $k \in \mathbb{Z}$;

(2) If $\ell \geq k$, then either $Q_{\gamma}^{\ell} \subseteq Q_{\beta}^{k}$ or $Q_{\beta}^{k} \cap Q_{\gamma}^{\ell}=\varnothing$;

(3) For arbitrary $(k, \beta)$ and for any $\ell \leq k$, there is a exclusive $\gamma$ such that $Q_{\beta}^{k} \subseteq Q_{\gamma}^{\ell}$; 
(4) For arbitrary $(k, \beta)$ there exists no more that $M$ (a settled geometric constant) $\gamma$ such that $Q_{\gamma}^{k+1} \subseteq Q_{\beta^{\prime}}^{k}$ and $Q_{\beta}^{k}=\cup_{Q \in \mathcal{D}_{k+1}, Q \subseteq Q_{\beta}^{k}} Q$;

(5) $B\left(x_{\beta}^{k}, a_{1} \delta^{k}\right) \subseteq Q_{\beta}^{k} \subseteq B\left(x_{\beta}^{k}, A_{1} \delta^{k}\right)=: B\left(Q_{\beta}^{k}\right)$;

(6) If $\ell \geq k$ and $Q_{\gamma}^{\ell} \subseteq Q_{\beta}^{k}$, then $B\left(Q_{\gamma}^{\ell}\right) \subseteq B\left(Q_{\beta}^{k}\right)$. The set $Q_{\beta}^{k}$ is called a dyadic cube of generation $k$ with centre $x_{\beta}^{k} \in Q_{\beta}^{k}$ and side length $\ell\left(Q_{\beta}^{k}\right)=\delta^{k}$.

From the natures of the dyadic system above, for any $Q_{\beta^{\prime}}^{k} Q_{\gamma}^{k+1}$ and $Q_{\gamma}^{k+1} \subset Q_{\beta^{\prime}}^{k}$, we get that there is a constant $\widetilde{A}_{0}>0$ such that:

$$
\left|Q_{\gamma}^{k+1}\right| \leq\left|Q_{\beta}^{k}\right| \leq \widetilde{A}_{0}\left|Q_{\gamma}^{k+1}\right| .
$$

\subsection{Adjacent Systems of Dyadic Cubes}

Let $\left\{\mathcal{D}^{t}: t=1,2, \ldots, \mathcal{T}\right\}$ be a limited set of the dyadic families, then we call that it is a collection of neighbor systems of dyadic cubes with arguments $\delta \in(0,1), 0<a_{1} \leq A_{1}<\infty$ and $1 \leq C_{a d j}<\infty$ if it has the following two characteristics:

(1) For any $t \in\{1,2, \ldots, \mathcal{T}\}, \mathcal{D}^{t}$ is a system of dyadic cubes with arguments $\delta \in(0,1)$ and $0<a_{1} \leq A_{1}<\infty$;

(2) For any ball $B(x, r) \subseteq \mathbb{H}$ with $\delta^{k+3}<r \leq \delta^{k+2}, k \in \mathbb{Z}$, there have $t \in\{1,2, \ldots, \mathcal{T}\}$ and $Q \in \mathcal{D}^{t}$ of generation $k$ which is centered on ${ }^{t} x_{\beta}^{k}$ such that $d\left(x,{ }^{t} x_{\beta}^{k}\right)<2 A_{0} \delta^{k}$ and

$$
B(x, r) \subseteq Q \subseteq B\left(x, C_{a d j} r\right)
$$

\subsection{Sparse Operators}

We review the concept of sparse family given in [12] on ordinary spaces of homogeneous description in the sense of Coifman and Weiss [13], which is also suitable in the case of homogeneous groups.

Definition 5. Let $0<\eta<1$, for every $Q \in \mathcal{S}$, we call that the collection $\mathcal{S} \subset \mathcal{D}$ of dyadic cubes be a $\eta$-sparse, if there exists a measurable subset $E_{Q} \subset Q$ such that $\left|E_{Q}\right| \geq \eta|Q|$ and the sets $\left\{E_{Q}\right\}_{Q \in \mathcal{S}}$ have only limited overlap.

Definition 6. Given a sparse family, the sparse operator $\mathcal{A}_{\mathcal{S}}$ is defined by

$$
\mathcal{A}_{\mathcal{S}}(f)(x)=\sum_{Q \in \mathcal{S}}\langle f\rangle_{Q} \chi_{Q}(x)
$$

where $\langle f\rangle_{Q}=\frac{1}{|Q|} \int_{Q} f(x) d x$.

In this subfraction, the primary target is to reveal the following quantitative edition of Lacey's pointwise domination inequality.

Proposition 1. Let $0<\alpha<N$. Let $m$ be a nonnegative integer. For every $f \in C_{c}^{\infty}(\mathbb{H})$ and $b \in L_{\text {loc }}^{m}(\mathbb{H})$, there exits $\mathcal{T}$ dyadic systems $\mathcal{D}^{t}, t=1,2, \ldots, \mathcal{T}$ and $\eta$-sparse families $\mathcal{S}_{t} \subset \mathcal{D}^{t}$ such that for a.e. $x \in \mathbb{H}$,

$$
\left|\left(I_{\alpha}\right)_{b}^{m} f\right| \leq C_{N, m, \alpha} \sum_{t=1}^{\mathcal{T}} \sum_{k=0}^{m}\left(\begin{array}{c}
m \\
k
\end{array}\right) \mathcal{A}_{\alpha, \mathcal{S}_{t}}^{m, k}(b, f)(x), \quad \text { a.e. } x \in \mathbb{H},
$$

where for a sparse family $\mathcal{S}, \mathcal{A}_{\alpha, \mathcal{S}}^{m, k}(b, \cdot)$ is the sparse operator given by

$$
\mathcal{A}_{\alpha, \mathcal{S}}^{m, k}(b, f)(x)=\sum_{Q \in \mathcal{S}}\left|b(x)-b_{Q}\right|^{m-k}|Q|^{\stackrel{\alpha}{N}}\left\langle f\left(b-b_{Q}\right)^{k}\right\rangle_{Q} \chi_{Q}(x) .
$$


To show the Proposition 1, we need some auxiliary maximal operators. To begin with, let $\widetilde{j_{0}}$ be the smallest integer such that

$$
2^{\tilde{j_{0}}}>\max \left\{3 A_{0}, 2 A_{0} C_{a d j}\right\}
$$

and let $C_{\widetilde{j_{0}}}:=2^{\widetilde{j_{0}}+2} A_{0}$.

Next we define the grand maximal truncated operator $\mathcal{M}_{I_{\alpha}}$ as follows:

$$
\mathcal{M}_{I_{\alpha}} f(x)=\sup _{x \in B} \operatorname{ess} \sup _{\xi \in B}\left|I_{\alpha}\left(f \chi_{\mathbb{H} \backslash \backslash C_{\tilde{j_{0}}} B}\right)(\xi)\right|,
$$

where the first supremum is taken over of all balls $B \subset \mathbb{H}$ satisfying $x \in B$. We can know that this operator is of vital importance in the following proof, Given a ball $B_{0} \subset \mathbb{H}$, for $x \in B_{0}$ we also define a local edition of $\mathcal{M}_{I_{\alpha}}$ by

$$
\mathcal{M}_{I_{\alpha}, B_{0}} f(x)=\sup _{x \in B \subset B_{0}} \operatorname{esssup}\left|I_{\alpha \in B}\left(f \chi_{C_{\tilde{j_{0}}} B_{0} \backslash C_{\tilde{j_{0}}} B}\right)(\xi)\right| .
$$

Now, we claim that the following lemma is true.

Lemma 1. Let $0<\alpha<N$. The following pointwise estimates holds:

1. For a.e. $x \in B_{0}$,

$$
\left|I_{\alpha}\left(f \chi_{{\tilde{j_{0}}}_{\tilde{j}_{0}}}\right)(x)\right| \leq \mathcal{M}_{I_{\alpha}, B_{0}} f(x) .
$$

2. There exists a constant $C_{N, \alpha}>0$ such that for a.e. $x \in \mathbb{H}$,

$$
\mathcal{M}_{I_{\alpha}} f(x) \leq C_{N, \alpha}\left(M_{\alpha} f(x)+I_{\alpha}|f|(x)\right) .
$$

Using the results of Lemma 1, we then prove the Proposition 1.

Proof of Proposition 1. In order to proof the Proposition 1, we refer to the thinking in [8] for this domination, which is adapted to our situation of homogeneous groups.

Firstly, we suppose that $f$ is supported in a ball $B_{0}:=B\left(x_{0}, r\right) \subset \mathbb{H}$, next we disintegrate $\mathbb{H}$ which respect to this ball $B_{0}$. We can do it as follows. We start define the annuli $U_{j}:=2^{j+1} B_{0} \backslash 2^{j} B_{0}, j \geq 0$ and select the minimum integer $j_{0}$ such that

$$
j_{0}>\tilde{j_{0}} \quad \text { and } \quad 2^{j_{0}}>4 A_{0}
$$

Next, for any $U_{j}$, we select the balls

$$
\left\{\widetilde{B}_{j, \ell}\right\}_{\ell-1^{\prime}}^{L_{j}}
$$

centred in $U_{j}$ and with radius $2^{j-\tilde{j}_{0}} r$ to cover $U_{j}$. From the doubling property [13], we obtain

$$
\sup _{j} L_{j} \leq C_{A_{0}, \tilde{j_{0}}}
$$

where $C_{A_{0}, \widetilde{j_{0}}}$ is an positive constant that only relates on $A_{0}$ and $\widetilde{j_{0}}$.

We now go over the characters of these $\widetilde{B}_{j, \ell}$. Denote $\widetilde{B}_{j, \ell}:=B\left(x_{j, \ell}, 2^{j-\widetilde{j}_{0}} r\right)$, where $\widetilde{j}_{0}$ is defines as in (4). Then we have $C_{a d j} \widetilde{B}_{j, \ell}:=B\left(x_{j, \ell}, C_{a d j} 2^{j-\widetilde{j}_{0}} r\right)$, which was shown in the proof of Theorem 3.7 in [12] that

$$
C_{a d j} \widetilde{B}_{j, \ell} \cap U_{j+j_{0}}=\varnothing, \quad \forall j \geq 0 \text { and } \forall \ell=1,2, \ldots, L_{j} ;
$$


and

$$
C_{a d j} \widetilde{B}_{j, \ell} \cap U_{j-j_{0}}=\varnothing, \quad \forall j \geq j_{0} \quad \text { and } \quad \forall \ell=1,2, \ldots, L_{j} .
$$

Now, because of the Equation (8) and (9), we see that each $C_{a d j} \widetilde{B}_{j, \ell}$, at most overlap with $2 j_{0}+1$ annuli $U_{j}$ 's. Moreover, for every $j$ and $\ell, C_{\widetilde{j_{0}}} \widetilde{B}_{j, \ell}$ covers $B_{0}$.

Next by observing the (2), there is an integer $t_{0} \in\{1,2, \ldots, \mathcal{T}\}$ and $Q_{0} \in \mathcal{D}^{t_{0}}$ such that $B_{0} \subseteq Q_{0} \subseteq C_{a d j} B_{0}$. Additionally, for this $Q_{0}$, as in Section 2.1 the ball that includes $Q_{0}$ and has comparable measure to $Q_{0}$ is represented by $B\left(Q_{0}\right)$. Consequently, $B_{0}$ is overwritten by $B\left(Q_{0}\right)$ and $\left|B\left(Q_{0}\right)\right| \lesssim\left|B_{0}\right|$, where the implicit constant relates only to $C_{a d j}$ and $A_{1}$.

Now we claim that there exists a $\frac{1}{2}$-sparse family $\mathcal{F}^{t_{0}} \subset \mathcal{D}^{t_{0}}\left(Q_{0}\right)$, the set of all dyadic cubes in $t_{0}$-th dyadic system that are contained in $Q_{0}$, such that for a.e. $x \in B_{0}$,

$$
\left|\left(I_{\alpha}\right)_{b}^{m}\left(f \chi_{C_{\tilde{j_{0}}} B\left(Q_{0}\right)}\right)(x)\right| \leq C_{N, m, \alpha} \sum_{k=0}^{m}\left(\begin{array}{c}
m \\
k
\end{array}\right) \mathcal{B}_{\alpha, \mathcal{F}^{t_{0}}}^{m,{ }^{\prime}}(b, f)(x),
$$

where

$$
\mathcal{B}_{\alpha, \mathcal{F}^{t_{0}}}^{m, k}(b, f)(x)=\sum_{Q \in \mathcal{F}^{t_{0}}}\left|b(x)-b_{R_{Q}}\right|^{m-k}\left|C_{\tilde{j}_{0}} B(Q)\right|^{\stackrel{\alpha}{N}}\left\langle f\left(b-b_{R_{Q}}\right)^{k}\right\rangle_{C_{\tilde{j}_{0}} B(Q)} \chi_{Q}(x) .
$$

Here, $R_{Q}$ is the dyadic cube in $\mathscr{D}^{t}$ for some $t \in\{1,2, \ldots, \mathcal{T}\}$ such that $C_{\tilde{j}_{0}} B(Q) \subset$ $R_{Q} \subset C_{a d j} \cdot C_{\tilde{j}_{0}} B(Q)$, where $B(Q)$ is defined as in Section 2.1, $j_{0}$ defined as in (5) and $\widetilde{j_{0}}$ defined as in (4).

Assume that we have already proven the assertion (10). Let us take a partition of $\mathbb{H}$ as follows:

$$
\mathbb{H}=\bigcup_{j=0}^{\infty} 2^{j} B_{0}
$$

We next consider the annuli $U_{j}:=2^{j+1} B_{0} \backslash 2^{j} B_{0}$ for $j \geq 0$ and the covering $\left\{\widetilde{B}_{j, \ell}\right\}_{\ell=1}^{L_{j}}$ of $U_{j}$ as in (6). We note that for each $\widetilde{B}_{j, \ell}$, there exist $t_{j, \ell} \in\{1,2, \ldots, \mathcal{T}\}$ and $\widetilde{Q}_{j, \ell} \in \mathcal{D}^{t_{j, \ell}}$ such that $\widetilde{B}_{j, \ell} \subseteq \widetilde{Q}_{j, \ell} \subseteq C_{a d j} \widetilde{B}_{j, \ell}$. Therefore, we acquire that for each such $\widetilde{B}_{j, \ell}$, the enlargement $C_{\widetilde{j_{0}}} B\left(\widetilde{Q}_{j, l}\right)$ covers $B_{0}$ since $C_{\widetilde{j_{0}}} \widetilde{B}_{j, \ell}$ covers $B_{0}$.

Next, we utilize (10) to each $\widetilde{B}_{j, \ell}$, then we acquire a $\frac{1}{2}$-sparse family $\widetilde{\mathcal{F}}_{j, \ell} \subset \mathcal{D}^{t_{j, \ell}}\left(\widetilde{Q}_{j, \ell}\right)$ such that (10) can be established for a.e. $x \in \widetilde{B}_{j, \ell}$.

Now, set $\mathcal{F}:=\cup_{j, \ell} \widetilde{\mathcal{F}}_{j, \ell}$. Then we observe that the balls $C_{a d j} \widetilde{B}_{j, \ell}$ are overlapping not more than $C_{A_{0}, \tilde{j} \tilde{j}_{0}}\left(2 j_{0}+1\right)$ times, where $C_{A_{0}, \tilde{j_{0}}}$ is the constant in (7). Then, we can obtain that $\mathcal{F}$ is a $\frac{1}{2 C_{A_{0}, \tilde{j} 0}\left(2 j_{0}+1\right)}$-sparse family and for a.e. $c \in \mathbb{H}$,

$$
\begin{aligned}
& \left|\left(I_{\alpha}\right)_{b}^{m}(f)(x)\right| \\
\leq & C_{N, m, \alpha} \sum_{k=0}^{m}\left(\begin{array}{c}
m \\
k
\end{array}\right) \sum_{Q \in \mathcal{F}}\left(\left|b(x)-b_{R_{Q}}\right|^{m-k}\left|C_{\tilde{j}_{0}} B(Q)\right|^{\stackrel{\alpha}{N}}\left\langle f\left(b-b_{R_{Q}}\right)^{k}\right\rangle_{\tilde{j}_{0}} B(Q)\right) \chi_{Q}(x) .
\end{aligned}
$$

Since $C_{\tilde{j}_{0}} B(Q) \subset R_{Q}$, and it is clear that $\left|R_{Q}\right| \leq \bar{C}\left|C_{\widetilde{j_{0}}} B(Q)\right|(\bar{C}$ depends only on $\left.C_{a d j}\right)$, we obtain that $\langle f\rangle_{C_{\tilde{j}} B(Q)} \leq \bar{C}\langle f\rangle_{R_{Q}}$. Now, we set $\mathcal{S}_{t}:=\left\{R_{Q} \in \mathcal{D}^{t}: Q \in \mathcal{F}\right\}, t \in$ $\{1,2, \ldots, \mathcal{T}\}$, then since the fact that $\mathcal{F}$ is $\frac{1}{2 C_{A_{0}, \tilde{j}_{0}}\left(2 j_{0}+1\right)}$-sparse, we can acquire that each family $\mathcal{S}_{t}$ is $\frac{1}{2 C_{A_{0}, \tilde{j} 0}\left(2 j_{0}+1\right) \bar{c}}$-sparse. 
Now, we let

$$
\eta:=\frac{1}{2 C_{A_{0}, \tilde{j_{0}}}\left(2 j_{0}+1\right) \overline{\bar{c}}}
$$

where $\bar{c}$ is a constant relating only on $\bar{C}, C_{\widetilde{j_{0}}}$. Then it follows that (3) holds, which finishes the proof.

Proof of the Assertion (10). To demonstrate the assertion it suffice to attest the following recursive computation: there exist the cubes $P_{j} \in \mathcal{D}^{t_{0}}\left(Q_{0}\right)$ that does not intersect each other such that $\sum_{j}\left|P_{j}\right| \leq \frac{1}{2}\left|Q_{0}\right|$ and for a.e. $x \in B_{0}$,

$$
\begin{aligned}
& \left|\left(I_{\alpha}\right)_{b}^{m}\left(f \chi_{C_{\tilde{j_{0}}} B\left(Q_{0}\right)}\right)(x)\right| \chi_{Q_{0}}(x) \\
\leq & C_{N, m, \alpha} \sum_{k=0}^{m}\left(\begin{array}{c}
m \\
k
\end{array}\right)\left|b(x)-b_{R_{Q_{0}}}\right|^{m-k}\left|C_{\tilde{j_{0}}} B\left(Q_{0}\right)\right|^{\frac{\alpha}{N}}\left\langle f\left(b-b_{R_{Q_{0}}}\right)^{k}\right\rangle_{C_{\tilde{j_{0}}} B\left(Q_{0}\right)} \chi_{Q_{0}}(x) \\
+ & \sum_{j}\left|\left(I_{\alpha}\right)_{b}^{m}\left(f \chi_{C_{\tilde{j_{0}}} B\left(P_{j}\right)}\right)(x)\right| \chi_{P_{j}}(x) .
\end{aligned}
$$

Iterating this estimate, we acquire (10) with $\mathcal{F}^{t_{0}}$ being the union of all the families $\left\{P_{j}^{k}\right\}$, where $\left\{P_{j}^{0}\right\}=\left\{Q_{0}\right\},\left\{P_{j}^{1}\right\}=\left\{P_{j}\right\}$ as mentioned above, and $\left\{P_{j}^{k}\right\}$ are the cubes acquired at the $k$-th stage of the iterative approach. Clearly $\mathcal{F}^{t_{0}}$ is a $\frac{1}{2}$-sparse family, since let

$$
E_{P_{j}^{k}}=P_{j}^{k} \backslash \cup_{j} P_{j}^{k+1} .
$$

Now we prove the recursive estimate. For any countable family $\left\{P_{j}\right\}_{j}$ of disjoint cubes $P_{j} \subset \mathcal{D}^{t_{0}}\left(Q_{0}\right)$, we have that

$$
\begin{aligned}
& \mid\left(I_{\alpha}\right)_{b}^{m}\left(f \chi_{C_{\tilde{j_{0}}} B\left(Q_{0}\right)}\right)(x) \chi_{Q_{0}}(x) \\
\leq & \left|\left(I_{\alpha}\right)_{b}^{m}\left(f \chi_{C_{\tilde{j_{0}}} B\left(Q_{0}\right)}\right)(x) \chi_{Q_{0} \backslash \cup_{j} P_{j}}(x)+\sum_{j}\right|\left(I_{\alpha}\right)_{b}^{m}\left(f \chi_{C_{\tilde{j_{0}}} B\left(Q_{0}\right)}\right)(x) \chi_{P_{j}}(x) \\
\leq & \left|\left(I_{\alpha}\right)_{b}^{m}\left(f \chi_{C_{\tilde{j_{0}}} B\left(Q_{0}\right)}\right)(x) \chi_{Q_{0} \backslash \cup_{j} P_{j}}(x)+\sum_{j}\right|\left(I_{\alpha}\right)_{b}^{m}\left(f \chi_{C_{\tilde{j_{0}}} B\left(Q_{0}\right) \backslash C_{\tilde{j_{0}}} B\left(P_{j}\right)}\right)(x) \chi_{P_{j}}(x) \\
+ & \sum_{j} \mid\left(I_{\alpha}\right)_{b}^{m}\left(f \chi_{C_{\tilde{j} 0} B\left(P_{j}\right)}\right)(x) \chi_{P_{j}}(x)
\end{aligned}
$$

So we just have to reveal that we can opt for a family of pairwise disjoint cubes $\left\{P_{j}\right\} \subset \mathcal{D}^{t_{0}}\left(Q_{0}\right)$ such that $\sum_{j}\left|P_{j}\right| \leq \frac{1}{2}\left|Q_{0}\right|$ and that for a.e. $x \in B_{0}$,

$$
\begin{aligned}
& \left|\left(I_{\alpha}\right)_{b}^{m}\left(f \chi_{C_{\tilde{j_{0}}} B\left(Q_{0}\right)}\right)(x)\right| \chi_{Q_{0} \backslash \cup_{j} P_{j}}(x)+\sum_{j}\left|\left(I_{\alpha}\right)_{b}^{m}\left(f \chi_{C_{\tilde{j_{0}}} B\left(Q_{0}\right) \backslash C_{\tilde{j_{0}}} B\left(P_{j}\right)}\right)(x)\right| \chi_{P_{j}}(x) \\
\leq & C_{N, m, \alpha} \sum_{k=0}^{m}\left(\begin{array}{c}
m \\
k
\end{array}\right)\left|b(x)-b_{R_{Q_{0}}}\right| m-k\left|C_{\tilde{j_{0}}} B\left(Q_{0}\right)\right|^{\stackrel{\alpha}{N}}\left\langle f\left(b-b_{R_{Q_{0}}}\right)^{k}\right\rangle_{C_{\tilde{j_{0}}} B\left(Q_{0}\right)} \chi_{Q_{0}}(x) .
\end{aligned}
$$

Using that $\left(I_{\alpha}\right)_{b}^{m} f=\left(I_{\alpha}\right)_{b-c}^{m} f$ for any $c \in \mathbb{R}$, and also that

$$
\left(I_{\alpha}\right)_{b-c}^{m} f=\sum_{k=0}^{m}(-1)^{k}\left(\begin{array}{c}
m \\
k
\end{array}\right) I_{\alpha}\left((b-c)^{k} f\right)(b-c)^{m-k},
$$


it follows that

$$
\begin{aligned}
& \left|\left(I_{\alpha}\right)_{b}^{m}\left(f \chi_{C_{\tilde{\tilde{j}_{0}}} B\left(Q_{0}\right)}\right)(x)\right| \chi_{Q_{0} \backslash \cup_{j} P_{j}(x)}+\sum_{j}\left|\left(I_{\alpha}\right)_{b}^{m}\left(f \chi_{C_{\tilde{\tilde{j}_{0}}} B\left(Q_{0}\right) \backslash C_{\tilde{j_{0}}} B\left(P_{j}\right)}\right)(x)\right| \chi_{P_{j}}(x) \\
\leq & \sum_{k=0}^{m}\left(\begin{array}{c}
m \\
k
\end{array}\right)\left|b(x)-b_{R_{Q_{0}}}\right|^{m-k}\left|I_{\alpha}\left(\left(b-b_{R_{Q_{0}}}\right)^{k} f \chi_{C_{\tilde{j_{0}}} B\left(Q_{0}\right)}\right)(x)\right| \chi_{Q_{0} \backslash \cup_{j} P_{j}}(x) \\
+ & \sum_{k=0}^{m}\left(\begin{array}{c}
m \\
k
\end{array}\right)\left|b(x)-b_{R_{Q_{0}}}\right|^{m-k} \sum_{j}\left|I_{\alpha}\left(\left(b-b_{R_{Q_{0}}}\right)^{k} f \chi_{C_{\tilde{j_{0}}} B\left(Q_{0}\right) \backslash C_{\tilde{j_{0}}} B\left(P_{j}\right)}\right)(x)\right| \chi_{P_{j}}(x) \\
= & : W_{1}+W_{2} .
\end{aligned}
$$

Now we define the set $E=\cup_{k=0}^{m} E_{k}$, where

$$
E_{k}=\left\{x \in B_{0}: \mathcal{M}_{I_{\alpha}, B_{0}}\left(\left(b-b_{R_{Q_{0}}}\right)^{k} f\right)(x)>C_{N, m, \alpha}\left|C_{\tilde{j}_{0}} B\left(Q_{0}\right)\right|^{\stackrel{\alpha}{N}}\left\langle\left(b-b_{R_{Q_{0}}}\right)^{k} f\right\rangle_{C_{\tilde{j_{0}}} B\left(Q_{0}\right)}\right\},
$$

with $C_{N, m, \alpha}$ being a positive number to be chosen.

From [8], we can choose $C_{N, m, \alpha}$ big enough (depending on $C_{\widetilde{j_{0}}}, C_{a d j}$, and $A_{1}$ ) such that

$$
|E| \leq \frac{1}{4 \widetilde{A_{0}}}\left|B_{0}\right|
$$

where $\widetilde{A_{0}}$ is defined in Section 2.1. We now utilize the Calderón-Zygmund decomposition to the function $\chi_{E}$ on $B_{0}$ at the height $\lambda:=\frac{1}{2 \widetilde{A}_{0}}$, to acquire pairwise disjoint cubes $\left\{P_{j}\right\} \subset$ $\mathcal{D}^{t_{0}}\left(Q_{0}\right)$ such that

$$
\frac{1}{2 \widetilde{A}_{0}}\left|P_{j}\right| \leq\left|P_{j} \cap E\right| \leq \frac{1}{2}\left|P_{j}\right|
$$

and $\left|E \backslash \cup_{j} P_{j}\right|=0$. This implies that

$$
\sum_{j}\left|P_{j}\right| \leq \frac{1}{2}\left|B_{0}\right| \text { and } P_{j} \cap E^{\mathcal{c}} \neq \varnothing .
$$

Fix some $j$. Since we have $P_{j} \cap E^{c} \neq \varnothing$, we observe that

$$
\mathcal{M}_{I_{\alpha}, B_{0}}\left(\left(b-b_{R_{Q_{0}}}\right)^{k} f\right)(x) \leq C_{N, m, \alpha}\left|C_{\tilde{j_{0}}} B\left(Q_{0}\right)\right|^{\stackrel{\alpha}{N}}\left\langle\left(b-b_{R_{Q_{0}}}\right)^{k} f\right\rangle_{C_{\tilde{j_{0}}} B\left(Q_{0}\right)^{\prime}}
$$

which allows us to control the summation in $W_{2}$ by considering the cube $P_{j}$.

Now by (i) in Lemma 1, we know that

$$
\left|I_{\alpha}\left(\left(b-b_{R_{Q_{0}}}\right)^{k} f \chi_{C_{\tilde{j_{0}}} B\left(Q_{0}\right)}\right)(x)\right| \leq \mathcal{M}_{I_{\alpha}, B_{0}}\left(\left(b-b_{R_{Q_{0}}}\right)^{k} f\right)(x), \quad \text { for a.e. } x \in B_{0} .
$$

Since $\left|E \backslash \cup_{j} P_{j}\right|=0$, we have that

$$
\begin{aligned}
& \mathcal{M}_{I_{\alpha}, B_{0}}\left(\left(b-b_{R_{Q_{0}}}\right)^{k} f\right)(x) \\
\leq & C_{N, m, \alpha}\left|C_{\tilde{j_{0}}} B\left(Q_{0}\right)\right|^{\frac{\alpha}{N}}\left\langle\left(b-b_{R_{Q_{0}}}\right)^{k} f\right\rangle_{C_{\tilde{j_{0}}} B\left(Q_{0}\right)^{\prime}} \quad \text { for a.e. } x \in B_{0} \backslash \cup_{j} P_{j} .
\end{aligned}
$$

Consequently,

$$
\begin{aligned}
& \left|I_{\alpha}\left(\left(b-b_{R_{Q_{0}}}\right)^{k} f \chi_{C_{\tilde{j_{0}}} B\left(Q_{0}\right)}\right)(x)\right| \\
\leq & C_{N, m, \alpha}\left|C_{\tilde{j}_{0}} B\left(Q_{0}\right)\right|^{\frac{\alpha}{N}}\left\langle\left(b-b_{R_{Q_{0}}}\right)^{k} f\right\rangle_{C_{\tilde{j_{0}}} B\left(Q_{0}\right)^{\prime}} \quad \text { for a.e. } x \in B_{0} \backslash \cup_{j} P_{j} .
\end{aligned}
$$


These estimates allow us to control the remaining terms in $W_{1}$, so we are done.

Proof of Lemma 1. Now we give the proof process of Lemma 1.

The result in the Euclidean space case can be referred to as [8]. Now, we can adapt the proof in [8] to our setting of homogeneous groups.

(i) Let $r$ is close enough to 0 such that $B(x, r) \subset B_{0}$. Then,

$$
\begin{aligned}
\left|I_{\alpha}\left(f \chi_{C_{\tilde{j_{0}}} B_{0}}\right)(x)\right| & \leq\left|I_{\alpha}\left(f \chi_{C_{\tilde{j_{0}}} B(x, r)}\right)(x)\right|+\left|I_{\alpha}\left(f \chi_{C_{\tilde{j_{0}}} B_{0} \backslash C_{\tilde{j_{0}}} B(x, r)}\right)(x)\right| \\
& \leq\left|I_{\alpha}\left(f \chi_{C_{\tilde{\tilde{j}_{0}}} B(x, r)}\right)(x)\right|+\mathcal{M}_{I_{\alpha}, B_{0}} f(x),
\end{aligned}
$$

the estimate for the first term follows by standard computations involving a dyadic annulitype decomposition of the $B(x, r)$.

$$
\begin{aligned}
\left|I_{\alpha}\left(f \chi_{C_{\tilde{j_{0}}} B(x, r)}\right)(x)\right| & =\left|\int_{\mathbb{H}} \frac{f(y) \chi_{C_{\tilde{j_{0}}} B(x, r)}}{d(x, y)^{N-\alpha}} d y\right| \\
& \leq \int_{B\left(x, C_{\tilde{j_{0}}} r\right)} \frac{|f(y)|}{d(x, y)^{N-\alpha}} d y \\
& =\sum_{i=-\infty}^{1} \int_{B\left(x, C_{\tilde{j}_{0}}{ }^{i}\right) \backslash B\left(x, C_{\tilde{j_{0}}}^{i-1} r\right)} \frac{|f(y)|}{d(x, y)^{N-\alpha}} d y \\
& \leq \sum_{i=-\infty}^{1}\left(C_{\tilde{j}_{0}}^{i-1} r\right)^{\alpha-N} \int_{B\left(x, C_{\tilde{j}_{0}}^{i} r\right)}|f(y)| d y \\
& =\sum_{i=-\infty}^{1}\left(\frac{1}{C_{\tilde{j_{0}}}}\right)^{\alpha-N}\left(C_{\tilde{j}_{0}}^{i} r\right)^{\alpha} \frac{1}{\left(C_{\tilde{j}_{0}}^{i} r\right)^{N}} \int_{B\left(x, C_{\tilde{j_{0}}}^{i} r\right)}|f(y)| d y \\
& \leq C_{N, \alpha, C_{\tilde{j}_{0}}} r^{\alpha} M f(x) .
\end{aligned}
$$

Then,

$$
\left|I_{\alpha}\left(f \chi_{C_{\tilde{j_{0}}} B_{0}}\right)(x)\right| \leq C_{N, \alpha, C_{\tilde{j_{0}}}} r^{\alpha} M f(x)+\mathcal{M}_{I_{\alpha}, B_{0}} f(x),
$$

the estimate in (i) is settled letting $r \rightarrow 0$ in (11).

(ii) Let $x, \xi \in B:=B\left(x_{0}, r\right)$. Let $B_{x}$ be the closed ball with radius $4\left(A_{0}+C_{\widetilde{j_{0}}}\right) r$, which centered at $x$. Then $C_{\widetilde{j_{0}}} B \subset B_{x}$, and we acquire

$$
\begin{aligned}
\left|I_{\alpha}\left(f \chi_{\mathbb{H} \backslash C_{\tilde{j_{0}}} B}\right)(\xi)\right| & =\left|I_{\alpha}\left(f \chi_{\mathbb{H} \backslash B_{x}}\right)(\xi)+I_{\alpha}\left(f \chi_{B_{x} \backslash C_{\tilde{j_{0}}} B}\right)(\xi)\right| \\
& \leq\left|I_{\alpha}\left(f \chi_{\mathbb{H} \backslash B_{x}}\right)(\xi)-I_{\alpha}\left(f \chi_{\mathbb{H} \backslash B_{x}}\right)(x)\right| \\
& +\left|I_{\alpha}\left(f \chi_{B_{x} \backslash C_{\tilde{\tilde{j}_{0}}} B}\right)(\xi)\right|+\left|I_{\alpha}\left(f \chi_{\mathbb{H} \backslash B_{x}}\right)(x)\right|
\end{aligned}
$$

For the first term, since $\rho$ is homogeneous of degree $\alpha-N$, and by using the Proposition 1.7 in [1], we get 


$$
\begin{aligned}
& \left|I_{\alpha}\left(f \chi_{\mathbb{H} \backslash B_{x}}\right)(\xi)-I_{\alpha}\left(f \chi_{\mathbb{H} \backslash B_{x}}\right)(x)\right| \\
\leq & \int_{\mathbb{H} \backslash B_{x}}|f(y)| \frac{1}{d(y, \xi)^{N-\alpha}}-\frac{1}{d(x, y)^{N-\alpha}} \mid d y \\
\leq & C_{N, \alpha} \int_{\mathbb{H} \backslash B_{x}} \frac{2 r}{d(x, y)^{N-\alpha+1}}|f(y)| d y \\
= & C_{N, \alpha} \sum_{i=1}^{\infty} \int_{2^{i} B_{x} \backslash 2^{i-1} B_{x}} \frac{2 r}{d(x, y)^{N-\alpha+1}}|f(y)| d y \\
\leq & C_{N, \alpha} \sum_{i=1}^{\infty} \frac{2 r}{\left(2^{i-1}\left|B_{x}\right|^{\frac{1}{N}}\right)^{N-\alpha+1}} \int_{2^{i} B_{x}}|f(y)| d y \\
= & C_{N, \alpha} \sum_{i=1}^{\infty} \frac{2 r}{\left(2^{i-1} 2^{2} r\left(A_{0}+C_{\tilde{j_{0}}}\right)\right)^{N-\alpha+1}} \int_{2^{i} B_{x}}|f(y)| d y \\
= & C_{N, \alpha} \sum_{i=1}^{\infty} \frac{2 r}{2^{i+1} r\left(A_{0}+C_{\widetilde{j_{0}}}\right)} \cdot \frac{1}{\left(2^{i+1} r\left(A_{0}+C_{\tilde{j_{0}}}\right)\right)^{N-\alpha}} \int_{2^{i} B_{x}}|f(y)| d y \\
\leq & C_{N, \alpha} M_{\alpha} f(x) .
\end{aligned}
$$

Next, for $\xi \in B, y \in B_{x} \backslash C_{\tilde{j_{0}}} B$, we have $d(y, \xi)>2^{\tilde{j_{0}}} r$. Then we have

$$
\begin{aligned}
\left|I_{\alpha}\left(f \chi_{B_{x} \backslash C_{\tilde{j_{0}}} B}\right)(\xi)\right| & \leq \int_{B_{x} \backslash C_{\tilde{j_{0}}} B} \frac{1}{d(y, \xi)^{N-\alpha}}|f(y)| d y \\
& \leq \frac{1}{\left|2^{\widetilde{j_{0}}} r\right|^{N-\alpha}} \int_{B_{x}}|f(y)| d y \\
& =C_{N, \alpha} \frac{1}{\left|4\left(A_{0}+C_{\widetilde{j_{0}}}\right) r\right|^{N-\alpha}} \int_{B_{x}}|f(y)| d y \\
& \leq C_{N, \alpha} M_{\alpha} f(x) .
\end{aligned}
$$

Finally, we observe that

$$
\begin{aligned}
\left|I_{\alpha}\left(f \chi_{\mathbb{H} \backslash B_{x}}\right)(x)\right| & =\left|\int_{\mathbb{H} \backslash B_{x}} \frac{f(y)}{d(x, y)^{N-\alpha}} d x\right| \\
& \leq \int_{\mathbb{H}} \frac{|f(y)|}{d(x, y)^{N-\alpha}} d x \\
& =I_{\alpha}|f|(x),
\end{aligned}
$$

which finishes the proof of (ii).

Next, we review that the dyadic weighted $B M O$ space associated with the system $\mathcal{D}^{t}$ is defined as

$$
B M O_{\eta, \mathcal{D}^{t}}(\mathbb{H}):=\left\{b \in L_{l o c}^{1}(\mathbb{H}):\|b\|_{B M O_{\eta, \mathcal{D}^{t}}}<\infty\right\},
$$

where $\|b\|_{B M O_{\eta, D^{t}}}=\sup _{Q \in \mathcal{D}^{t}} \frac{1}{\eta(Q)} \int_{Q}\left|b(x)-b_{Q}\right| d x$. Then according to the dyadic structure theorem studies in [14], one has

$$
B M O_{\eta}(\mathbb{H})=\bigcap_{t=1}^{\mathcal{T}} B M O_{\eta, \mathcal{D}^{t}}(\mathbb{H}) .
$$


Now, to verify a function $b$ is in $B M O_{\eta}(\mathbb{H})$, it suffices to verify it belongs to each weighted dyadic $B M O$ space $B M O_{\eta, D^{t}}(\mathbb{H})$. Given a dyadic cube $Q \in \mathcal{D}^{t}$ with $t=$ $1,2, \ldots, \mathcal{T}$, and a measurable function $f$ on $\mathbb{H}$, we define the local mean oscillation of $f$ on $Q$ by

$$
\omega_{\lambda}(f ; Q)=\inf _{c \in \mathbb{R}}\left((f-c) \chi_{Q}\right)^{*}(\lambda|Q|), \quad 0<\lambda<1,
$$

where

$$
\left((f-c) \chi_{Q}\right)^{*}(\lambda|Q|)=\sup _{E \subset Q,|E|=\lambda|Q|} \inf _{x \in E}|(f-c)(x)| .
$$

With these notation and dyadic structure theorem above, following the same proof in [10], we also acquire that for any weight $\eta \in A_{2}$, we have

$$
\|b\|_{B M O_{\eta}(\mathbb{H})} \leq C \sum_{t=1}^{\mathcal{T}} \sup _{Q \in \mathcal{D}^{t}} \omega_{\lambda}(b ; Q) \frac{|Q|}{\eta(Q)}, \quad 0<\lambda \leq 2^{N+1},
$$

where $C$ depends on $\eta$.

Proposition 2. Suppose that $\mathbb{H}$ is a homogeneous group with dimension $N, b \in L_{\text {loc }}^{1}(\mathbb{H})$. Then for any cube $Q \subset \mathbb{H}$, there exist measurable set $F_{i} \subset Q$ with $i=1,2$, such that

$$
\omega_{2 \frac{1}{N+2}}(b ; Q) \leq b(x)-b(y), \forall(x, y) \in F_{1} \times F_{2} .
$$

Proof. We take ideas from N. Accomazzo, J. C. Martínez-Perales and I. P. Rivera-Ríos [8]. In [8], for any cube $Q \in \mathcal{D}^{t}$ with $t=1,2, \ldots, \mathcal{T}$, there exists a subset $E \subset Q$ with $|E|=$ $\frac{1}{2^{N+2}}|Q|$ such that for every $x \in E$,

$$
\omega_{2 \frac{1}{N+2}}(b ; Q) \leq\left|b(x)-m_{b}(Q)\right|,
$$

where $m_{b}(Q)$ is a not necessarily unique number that satisfies

$$
\max \left\{\left|\left\{x \in Q: b(x)>m_{b}(Q)\right\}\right|,\left|\left\{x \in Q: b(x)<m_{b}(Q)\right\}\right|\right\} \leq \frac{|Q|}{2} .
$$

Let $E_{1} \subset Q$ with $|E|=\frac{1}{2}|Q|$ and such that $b(x) \geq m_{b}(Q)$ for every $x \in E_{1}$. Further let $E_{2}=Q \backslash E_{1}$, then $\left|E_{2}\right|=\frac{1}{2}|Q|$ and for every $x \in E_{2}, b(x) \leq m_{b}(Q)$.

We obtain that at least half of the set $E$ is contained either in $E_{1}$ or in $E_{2}$ since $Q$ is the disjoint union of $E_{1}$ and $E_{2}$. Without loss of generality, we assume that half of $E$ is in $E_{1}$, then we let $F_{1}=E \cap E_{1}, F_{2}=E_{2} \cap\left(E \cap E_{1}\right)^{c}$, we have

$$
\left|F_{1}\right|=|E|-\left|E \cap\left(E \cap E_{1}\right)^{C}\right| \geq|E|-\frac{|E|}{2}=\frac{|Q|}{2^{N+3}},
$$

and

$$
\left|F_{2}\right|=\left|E_{2}\right|-\left|E_{2} \cap\left(E \cap E_{1}\right)\right| \geq \frac{1}{2}|Q|-\frac{1}{2^{N+3}}|Q|=\left(\frac{1}{2}-\frac{1}{2^{N+3}}\right)|Q| .
$$

Then if $x \in F_{1}$ and $y \in F_{2}$, we have that

$$
\omega_{2^{\frac{1}{N+2}}}(b ; Q) \leq b(x)-m_{b}(Q) \leq b(x)-b(y),
$$

which shows that Proposition 2 holds. 
Given a dyadic grid $\mathcal{D}$, define the dyadic Riesz potential operator

$$
I_{\alpha}^{\mathcal{D}} f(x)=\sum_{Q \in \mathcal{D}} \frac{1}{|Q|^{1-\frac{\alpha}{N}}} \int_{Q}|f(y)| d y \chi_{Q}(x) .
$$

Proposition 3. Given $0<\alpha<N$, then for any dyadic grid $\mathcal{D}$,

$$
I_{\alpha}^{\mathcal{D}} f(x) \lesssim I_{\alpha} f(x)
$$

Proof. The result in the Euclidean setting is from the Proposition 2.1 in [15]. Here, we can adapt the proof in [15] to our setting of spaces of homogeneous type.

\section{Proof of Theorem 1}

To proof (i), we are following the ideas in [16] or [8].

Let $\mathcal{D}$ be a dyadic system in $\mathbb{H}$ and let $\mathcal{S}$ be a sparse family from $\mathcal{D}$. We know

$$
\mathcal{A}_{\alpha, \mathcal{S}}^{m, k}(b, f)(x)=\sum_{Q \in \mathcal{S}}\left|b(x)-b_{Q}\right|^{m-k}|Q|^{\frac{\alpha}{N}}\left\langle\left(b-b_{Q}\right)^{k} f\right\rangle_{Q} \chi_{Q}(x),
$$

by duality, we have that

$$
\begin{aligned}
\left\|\mathcal{A}_{\alpha, \mathcal{S}}^{m, k}(b, f)(x)\right\|_{L_{\lambda}^{q}(\mathbb{H})} \leq \sup _{g:\|g\|_{L_{\lambda q^{\prime}}^{q^{\prime}(\mathbb{H})}}} \sum_{Q \in \mathcal{Q}}\left(\int_{Q}\left|g(x) \lambda^{q}\right|\left|b(x)-b_{Q}\right|^{m-k} d x\right)|Q|^{\stackrel{\alpha}{N}} \\
\times\left(\frac{1}{|Q|} \int_{Q}\left|b(x)-b_{Q}\right|^{k}|f(x)| d x\right) .
\end{aligned}
$$

By Lemma 3.5 in [12], there exists a sparse family $\widetilde{\mathcal{S}} \subset \mathcal{D}$ such that $\mathcal{S} \subset \widetilde{\mathcal{S}}$ and for every cube $Q \in \widetilde{\mathcal{S}}$, for a.e. $x \in Q$,

$$
\left|b(x)-b_{Q}\right| \leq C_{N} \sum_{P \in \widetilde{\mathcal{S}}, P \subset Q} \Omega(b, P) \chi_{P}(x),
$$

where $\Omega(b, P)=\frac{1}{|P|} \int_{P}\left|b(x)-b_{P}\right| d x$

Assume that $b \in B M O_{\eta}(\mathbb{H})$ with $\eta$ to be chosen, then we have for a.e. $x \in Q$,

$$
\begin{aligned}
\left|b(x)-b_{Q}\right| & \leq C_{N} \sum_{P \in \widetilde{\mathcal{S}}, P \subset Q} \frac{1}{\eta(P)} \int_{P}\left|b(x)-b_{P}\right| d x \cdot \frac{\eta(P)}{|P|} \chi_{P}(x) \\
& \leq C_{N}\|b\|_{B M O_{\eta}}(\mathbb{H}) \sum_{P \in \widetilde{\mathcal{S}}, P \subset Q} \frac{\eta(P)}{|P|} \chi_{P}(x) .
\end{aligned}
$$

Then, we further have

$$
\begin{aligned}
& \left\|\mathcal{A}_{\alpha, \mathcal{S}}^{m, k}(b, f)(x)\right\|_{L_{\lambda}^{q}(\mathbb{H})} \\
& \leq C_{N}\|b\|_{B M O_{\eta}(\mathbb{H})}^{m} \sup _{g:\|g\|_{L_{\lambda q^{\prime}}^{q \prime}(\mathbb{H})}} \sum_{Q \in \mathcal{S}}\left(\frac{1}{|Q|} \int_{Q}\left|g(x) \lambda^{q}\right|\left(\sum_{P \in \widetilde{\mathcal{S}}, P \subset Q} \frac{\eta(P)}{|P|} \chi_{P}(x)\right)^{m-k} d x\right) \\
& \times\left(\frac{1}{|Q|} \int_{Q}\left(\sum_{P \in \widetilde{\mathcal{S}}, P \subset Q} \frac{\eta(P)}{|P|} \chi_{P}(x)\right)^{k}|f(x)| d x\right) \cdot|Q| \cdot|Q|^{\stackrel{\alpha}{N}} .
\end{aligned}
$$


Next, note that for each $\ell \in \mathbb{N}$, from [12], for an arbitrary function $h$, we have

$$
\begin{aligned}
& \int_{Q}|h(x)|\left(\sum_{Q \in \widetilde{\mathcal{S}}, P \subset Q} \frac{\eta(P)}{|P|} \chi_{P}(x)\right)^{\ell} d x \\
\lesssim & \int_{Q} \mathcal{A}_{\widetilde{\mathcal{S}}, \eta}^{\ell}(|h|)(x) d x,
\end{aligned}
$$

where $\mathcal{A}_{\widetilde{\mathcal{S}}, \eta}(|h|)(x):=\mathcal{A}_{\widetilde{\mathcal{S}}}(|h|) \eta, \mathcal{A}_{\widetilde{\mathcal{S}}}(h):=\sum_{Q \in \widetilde{\mathcal{S}}} h_{Q} \chi_{Q}$ and $\mathcal{A}_{\widetilde{\mathcal{S}}, \eta}^{\ell} f$ stands for the $\ell$-th iteration of $\mathcal{A}_{\widetilde{\mathcal{S}}, \eta}$.

Then we have

$$
\begin{aligned}
& \left\|\mathcal{A}_{\alpha, \mathcal{S}}^{m, k}(b, f)(x)\right\|_{L_{\lambda q}^{q}(\mathbb{H})} \\
& \leq C_{N}\|b\|_{B M O_{\eta}(\mathbb{H})}^{m} \sup _{g:\|g\|_{L_{\lambda q^{\prime}}^{q{ }^{\prime}}(\mathbb{H})}} \sum_{Q \in \mathcal{S}}\left(\int_{Q} \mathcal{A}_{\widetilde{\mathcal{S}}, \eta}^{m-k}\left(|g| \lambda^{q}\right)\right) \cdot \frac{1}{|Q|^{1-\frac{\alpha}{N}}}\left(\int_{Q} \mathcal{A}_{\widetilde{\mathcal{S}}, \eta}^{k}(|f|)\right) \\
& \leq C_{N}\|b\|_{B M O_{\eta}(\mathbb{H})}^{m} \sup _{g:\|g\|_{L_{\lambda q^{\prime}}^{q^{\prime}}(\mathbb{H})}=1} \int_{\mathbb{H}} \sum_{Q \in \mathcal{S}} \frac{1}{|Q|^{1-\frac{\alpha}{N}}}\left(\int_{Q} \mathcal{A}_{\widetilde{\mathcal{S}}, \eta}^{k}(|f|) \chi_{Q}(x)\right) \cdot \mathcal{A}_{\widetilde{\mathcal{S}}, \eta}^{m-k}\left(|g| \lambda^{q}\right) \\
& =C_{N}\|b\|_{B M O_{\eta}(\mathbb{H})}^{m} \sup _{g:\|g\|_{L_{\lambda q^{\prime}}^{q^{\prime}(\mathbb{H})}}=1} \int_{\mathbb{H}} I_{\mathcal{S}}^{\alpha}\left(\mathcal{A}_{\widetilde{\mathcal{S}}, \eta}^{k}(|f|)\right)(x)\left(\mathcal{A}_{\widetilde{\mathcal{S}}, \eta}^{m-k}\left(|g| \lambda^{q}\right)\right)(x) d x,
\end{aligned}
$$

where $I_{\mathcal{S}, \eta}^{\alpha} f:=I_{\mathcal{S}}^{\alpha}(f) \eta$ and $I_{\mathcal{S}}^{\alpha} f(x)=\sum_{Q \in \mathcal{S}} \frac{1}{|Q|^{1-\frac{\alpha}{N}}} \int_{Q}|f| \chi_{Q}(x)$.

From (13) and the boundedness of $I_{\alpha} f$, if $p, q, \alpha$ are as in the hypothesis of Theorem 1.1 and $w \in A_{p, q}, \mathcal{S} \subset \mathcal{D}$, then

$$
\left\|I_{\mathcal{S}}^{\alpha}\right\|_{L_{w p}^{p}(\mathbb{H}) \rightarrow L_{w q}^{q}(\mathbb{H})} \leq C_{N, p, q, \alpha}[w]_{A_{p, q}}^{\left(1-\frac{\alpha}{N}\right) \max \left\{1, \frac{p^{\prime}}{q}\right\}} .
$$

Observe that $\mathcal{A}_{\widetilde{\mathcal{S}}}$ is self-adjoint, then

$$
\int_{\mathbb{H}} I_{\mathcal{S}}^{\alpha}\left(\mathcal{A}_{\widetilde{\mathcal{S}}, \eta}^{k}(|f|)\right)\left(\mathcal{A}_{\widetilde{\mathcal{S}, \eta}}^{m-k}\left(|g| \lambda^{q}\right)\right)=\int_{\mathbb{H}} \mathcal{A}_{\widetilde{\mathcal{S}}} \mathcal{A}_{\widetilde{\mathcal{S}}, \eta}^{m-k-1}\left[I_{\mathcal{S}, \eta}^{\alpha}\left(\mathcal{A}_{\widetilde{\mathcal{S}}, \eta}^{K}(|f|)\right)\right]|g| \lambda^{q} .
$$

By Hölder inequality, we have that

$$
\left\|\mathcal{A}_{\alpha, \mathcal{S}}^{m, k}(b, f)(x)\right\|_{L_{\lambda q}^{q}(\mathbb{H})} \leq C_{N}\|b\|_{B M O_{\eta}(\mathbb{H})}^{m}\left\|\mathcal{A}_{\widetilde{\mathcal{S}}} \mathcal{A}_{\widetilde{\mathcal{S}}, \eta}^{m-k-1} I_{\mathcal{S}, \eta}^{\alpha} \mathcal{A}_{\widetilde{\mathcal{S}}, \eta}^{k}(|f|)\right\|_{L_{\lambda}^{q}(\mathbb{H})} .
$$

Applying that $\left\|\mathcal{A}_{\widetilde{\mathcal{S}}}\right\|_{L_{w}^{p}(\mathbb{H})} \leq C_{N, p}[w]_{A_{p}}^{\max \left\{1, \frac{1}{p-1}\right\}}$ (see, e.g., [17] ),

$$
\begin{aligned}
& \left\|\mathcal{A}_{\widetilde{\mathcal{S}}} \mathcal{A}_{\widetilde{\mathcal{S}}, \eta}^{m-k-1} I_{\mathcal{S}, \eta}^{\alpha} \mathcal{A}_{\widetilde{\mathcal{S}}, \eta}^{k}(|f|)\right\|_{L_{\lambda q}^{q}(\mathbb{H})} \\
\leq & C_{N, p}\left[\lambda^{q}\right]_{A_{q}}^{\max \left\{1, \frac{1}{q-1}\right\}}\left\|\mathcal{A}_{\widetilde{\mathcal{S}}} \mathcal{A}_{\widetilde{\mathcal{S}}, \eta}^{m-k-2} I_{\mathcal{S}, \eta}^{\alpha} \mathcal{A}_{\widetilde{\mathcal{S}}, \eta}^{k}(|f|)\right\|_{L_{\lambda \eta^{q}}^{q}(\mathbb{H})} \\
\leq & C_{N, p}\left[\lambda^{q}\right]_{A_{q}}^{\max \left\{1, \frac{1}{q-1}\right\}}\left[\lambda^{q} \eta^{q}\right]_{A_{q}}^{\max \left\{1, \frac{1}{q-1}\right\}}\left\|\mathcal{A}_{\widetilde{\mathcal{S}}, \eta}^{m-k-2} I_{\mathcal{S}, \eta}^{\alpha} \mathcal{A}_{\widetilde{\mathcal{S}}, \eta}^{k}(|f|)\right\|_{L_{\lambda \eta^{q} \eta^{q}}^{q}(\mathbb{H})} \\
\leq & \mathcal{C}_{N, p}\left(\prod_{i=0}^{m-k-1}\left[\lambda^{q} \eta^{i q}\right]_{A_{q}}\right)^{\max \left\{1, \frac{1}{q-1}\right\}}\left\|I_{\mathcal{S}, \eta}^{\alpha} \mathcal{A}_{\widetilde{\mathcal{S}}, \eta}^{k}(|f|)\right\|_{L_{\lambda \eta^{q}(m-k-1) q}^{q}(\mathbb{H})} .
\end{aligned}
$$


Using (14), we have that

$$
\begin{aligned}
\left\|I_{\mathcal{S}, \eta}^{\alpha} \mathcal{A}_{\widetilde{\mathcal{S}}, \eta}^{k}(|f|)\right\|_{L_{\lambda \eta_{\eta}(m-k-1) q}^{q}(\mathbb{H})} & =\left\|I_{\mathcal{S}}^{\alpha} \mathcal{A}_{\widetilde{\mathcal{S}}, \eta}^{k}(|f|)\right\|_{L_{\lambda \eta_{\eta}(m-k) q}^{q}(\mathbb{H})} \\
& \leq C_{N, p, \alpha}\left[\lambda \eta^{m-k}\right]_{A_{p, q}}^{\left(1-\frac{\alpha}{N}\right) \max \left\{1, \frac{p^{\prime}}{q}\right\}}\left\|\mathcal{A}_{\widetilde{\mathcal{S}}, \eta}^{k}(|f|)\right\|_{L_{\lambda p_{\eta}(m-k) p}^{p}(\mathbb{H})},
\end{aligned}
$$

and applying again $\left\|\mathcal{A}_{\widetilde{\mathcal{S}}}\right\|_{L_{w}^{p}(\mathbb{H})} \leq C_{N, p}[w]_{A_{p}}^{\max \left\{1, \frac{1}{p-1}\right\}}$

$$
\left\|\mathcal{A}_{\widetilde{\mathcal{S}}, \eta}^{k}(|f|)\right\|_{L_{\lambda \eta^{p}(m-k) p}^{p}(\mathbb{H})} \leq C_{N, p}\left(\prod_{i=m-k+1}^{m}\left[\lambda^{p} \eta^{i p}\right]_{A_{p}}\right)^{\max \left\{1, \frac{1}{p-1}\right\}}\|f\|_{L_{\lambda \eta^{m}}^{p}(\mathbb{H})^{\prime}}
$$

which, along with the previous estimate, yields

$$
\begin{aligned}
& \left\|\mathcal{A}_{\alpha, \mathcal{S}}^{m, k}(b, f)(x)\right\|_{L_{\lambda q}^{q}(\mathbb{H})} \\
\leq & C_{N, p, \alpha}\|b\|_{B M O_{\eta}(\mathbb{H})}^{m} A(m, k) B(m, k)\left[\lambda \eta^{m-k}\right]^{\left(1-\frac{\alpha}{N}\right) \max \left\{1, \frac{p^{\prime}}{q}\right\}}\|f\|_{L_{\lambda \eta^{m}}^{p} p(\mathbb{H})^{\prime}}
\end{aligned}
$$

where

$$
A(m, k)=\left(\prod_{i=0}^{m-k-1}\left[\lambda^{q} \eta^{i q}\right]_{A_{q}}\right)^{\max \left\{1, \frac{1}{q-1}\right\}}
$$

and

$$
B(m, k)=\left(\prod_{i=m-k+1}^{\infty}\left[\lambda^{p} \eta^{i p}\right]_{A_{p}}\right)^{\max \left\{1, \frac{1}{p-1}\right\}} .
$$

Hence, setting $\eta=v^{1 / m}$, where $v=\left(\frac{\mu}{\lambda}\right)^{1 / p}$, it reading follows from Hölder's inequality

$$
\left[\lambda^{s} v^{s \frac{i}{m}}\right]_{A_{s}} \leq\left[\lambda^{s}\right]_{A_{s}}^{\frac{m-i}{m}}\left[\mu^{s}\right]_{A_{s}}^{\frac{i}{m}}, \quad s=p, q .
$$

Thus, we acquire that

$$
A(m, k) \leq\left(\prod_{i=0}^{m-k-1}\left[\lambda^{q}\right]_{A_{q}}^{\frac{m-i}{m}}\left[\mu^{q}\right]_{A_{q}}^{\frac{i}{m}}\right)^{\max \left\{1, \frac{1}{q-1}\right\}} \leq\left(\left[\lambda^{q}\right]_{A_{q}}^{\frac{m+k+1}{2}}\left[\mu^{q}\right]_{A_{q}}^{\frac{m-k-1}{2}}\right)^{\frac{m-k}{m} \max \left\{1, \frac{1}{q-1}\right\}},
$$

and

$$
B(m, k) \leq\left(\prod_{i=m-k+1}^{m}\left[\lambda^{p}\right]_{A_{p}}^{\frac{m-i}{m}}\left[\mu^{p}\right]_{A_{p}}^{\frac{i}{m}}\right)^{\max \left\{1, \frac{1}{p-1}\right\}} \leq\left(\left[\lambda^{p}\right]_{A_{p}}^{\frac{k-1}{2}}\left[\mu^{p}\right]_{A_{p}}^{m-\frac{k-1}{2}}\right)^{\frac{k}{m} \max \left\{1, \frac{1}{p-1}\right\}} .
$$

Combining all the preceding estimates obtains (i).

To proof (ii), we are going to follow ideas in [10]. Based on (12), it suffices to show that there exists a positive constant $C$ such that for all dyadic cubes $Q \in \mathcal{D}^{t}$,

$$
\omega_{2 \frac{1}{N+2}}(b ; Q)^{m} \leq C\left(\frac{v^{1 / m}(Q)}{|Q|}\right)^{m}\left\|\left(I_{\alpha}\right)_{b}^{m}\right\|_{L_{\mu^{p}}^{p}(\mathbb{H}) \rightarrow L_{\lambda^{q}}^{q}(\mathbb{H})}
$$


Using Proposition 2 and Hölder inequality implies that

$$
\begin{aligned}
\omega_{2^{\frac{1}{N+2}}}(b ; Q)^{m}\left|F_{1}\right|\left|F_{2}\right| & \leq \int_{F_{1}} \int_{F_{2}}(b(x)-b(y))^{m} d x d y \\
& \leq \operatorname{dima}(Q)^{N-\alpha} \int_{F_{1}} \int_{F_{2}} \frac{(b(x)-b(y))^{m}}{d(x, y)^{N-\alpha}} d x d y \\
& =\operatorname{dima}(Q)^{N-\alpha} \int_{F_{1}}\left(I_{\alpha}\right)_{b}^{m}\left(\chi_{F_{2}}\right)(x) d x \\
& \leq C|Q|^{1-\frac{\alpha}{N}}\left(\int_{Q} \lambda^{-q^{\prime}}\right)^{\frac{1}{q^{\prime}}} \cdot\left(\int_{\mathbb{H}}\left[\left(I_{\alpha}\right)_{b}^{m}\left(\chi_{F_{2}}\right)\right]^{q} \lambda^{q} d x\right)^{\frac{1}{q}} \\
& \leq C|Q|^{1-\frac{\alpha}{N}}\left(\int_{Q} \lambda^{-q^{\prime}}\right)^{\frac{1}{q^{\prime}}} \cdot\left(\int_{Q} \mu^{p}\right)^{\frac{1}{p}}\left\|\left(I_{\alpha}\right)_{b}^{m}\right\|_{L_{\mu^{p}}^{p}(\mathbb{H}) \rightarrow L_{\lambda q}^{q}(\mathbb{H})} \\
& =C|Q|^{2}\left(\frac{1}{|Q|} \int_{Q} \lambda^{-q^{\prime}}\right)^{\frac{1}{q^{\prime}}} \cdot\left(\frac{1}{|Q|} \int_{Q} \mu^{p}\right)^{\frac{1}{p}}\left\|\left(I_{\alpha}\right)_{b}^{m}\right\|_{L_{\mu^{p}}^{p}(\mathbb{H}) \rightarrow L_{\lambda q}^{q}(\mathbb{H})^{\prime}}
\end{aligned}
$$

where we used that $\frac{1}{q}+\frac{\alpha}{N}=\frac{1}{p}$.

Further, this yields

$$
\omega_{2^{N+2}}(b ; Q)^{m} \leq C\left(\frac{1}{|Q|} \int_{Q} \lambda^{-q^{\prime}}\right)^{\frac{1}{q^{\prime}}} \cdot\left(\frac{1}{|Q|} \int_{Q} \mu^{p}\right)^{\frac{1}{p}}\left\|\left(I_{\alpha}\right)_{b}^{m}\right\|_{L_{\mu^{p}}^{p}(\mathbb{H}) \rightarrow L_{\lambda q}^{q}(\mathbb{H})} .
$$

Then from [8], we have

$$
\left(\frac{1}{|Q|} \int_{Q} \mu^{p}\right)^{\frac{1}{p}} \leq C\left(\frac{1}{|Q|} \int_{Q} v^{1 / m}\right)^{m}\left(\frac{1}{|Q|} \int_{Q} \lambda^{p}\right)^{\frac{1}{p}},
$$

so the

$$
\begin{aligned}
& \omega_{2^{\frac{1}{N+2}}}(b ; Q)^{m} \\
\leq & C\left(\frac{1}{|Q|} \int_{Q} v^{1 / m}\right)^{m}\left(\frac{1}{|Q|} \int_{Q} \lambda^{-q^{\prime}}\right)^{\frac{1}{q^{\prime}}}\left(\frac{1}{|Q|} \int_{Q} \lambda^{p}\right)^{\frac{1}{p}}\left\|\left(I_{\alpha}\right)_{b}^{m}\right\|_{L_{\mu^{p}}^{p}(\mathbb{H}) \rightarrow L_{\lambda^{q}}^{q}(\mathbb{H})} .
\end{aligned}
$$

Now we observe that since $q>p$ then by Hölder inequality,

$$
\left(\frac{1}{|Q|} \int_{Q} \lambda^{p}\right)^{\frac{1}{p}} \leq\left(\frac{1}{|Q|} \int_{Q} \lambda^{q}\right)^{\frac{1}{q}} \text { and }\left(\frac{1}{|Q|} \int_{Q} \lambda^{-q^{\prime}}\right)^{\frac{1}{q^{\prime}}} \leq\left(\frac{1}{|Q|} \int_{Q} \lambda^{-p^{\prime}}\right)^{\frac{1}{p^{\prime}}}
$$

then

$$
\left(\frac{1}{|Q|} \int_{Q} \lambda^{-q^{\prime}}\right)^{\frac{1}{q^{\prime}}}\left(\frac{1}{|Q|} \int_{Q} \lambda^{p}\right)^{\frac{1}{p}} \leq\left[\left(\frac{1}{|Q|} \int_{Q} \lambda^{q}\right)^{\frac{1}{q}}\left(\frac{1}{|Q|} \int_{Q} \lambda^{-p^{\prime}}\right)^{\frac{q}{p^{\prime}}}\right]^{\frac{1}{q}} .
$$

Consequently, since $\lambda \in A_{p, q}$, we finally get

$$
\omega_{2^{N+2}}(b ; Q)^{m} \leq C\left(\frac{1}{|Q|} \int_{Q} v^{1 / m}\right)^{m}\left\|\left(I_{\alpha}\right)_{b}^{m}\right\|_{L_{\mu^{p}}^{p}(\mathbb{H}) \rightarrow L_{\lambda q}^{q}(\mathbb{H})} .
$$

Thus, (15) holds and hence, the proof of (ii) is complete.

Therefore, we complete the proof of Theorem 1. 
Author Contributions: Writing—original draft preparation, D.C.; methodology, Y.C.; check, T.W. All authors have read and agreed to the published version of the manuscript.

Funding: The project was in part supported by: Yanping Chen's National Natural Sciience Foundation of China (\# 11871096, \# 11471033).

Institutional Review Board Statement: Not applicable.

Informed Consent Statement: Not applicable.

Data Availability Statement: Data sharing not applicable to this article as no datasets were generated or analysed during the current study.

Conflicts of Interest: The authors declare no conflict of interest.

\section{Abbreviation}

BMO Bounded Mean Oscillation

\section{References}

1. Folland, G.B.; Stein, E.M. Hardy Spaces on Homogeneous Groups; Princetion University Press: Princeton, NJ, USA; Univerity of Tokyo: Tokyo, Japan, 1982.

2. Stein, E.M. Harmonic Analysis: Real-Variable Methods, Orthogonality, and Oscillatory Integrals; Princeton University Press: Priceton, NJ, USA, 1993.

3. Frazier, M.; Jawerth, B.; Weiss, G. Littlewood-Paley Theory and the Study of Function Spaces; American Mathematical Society: Providence, RI, USA, 1991; Volume 79. [CrossRef]

4. Sawyer, E.; Wheeden, R.L. Weighted Inequalities for Fractional Integrals on Euclidean and Homogeneous Spaces. Am. J. Math. 1992, 114, 813-874. [CrossRef]

5. Muckenhoupt, B.; Wheeden, R. Weighted Norm Inequalities for Fractional Integrals. Trans. Am. Math. Soc. 1974, 192, 261-274. [CrossRef]

6. Lacey, M.T.; Moen, K.; Pérez, C.; Torres, R.H. Sharp Weighted Bounds for Fractional Integral Operators. J. Funct. Anal. 2010, 259, 1073-1097. [CrossRef]

7. Holmes, I.; Rahm, R.; Spencer, S. Commutators with Fractional Integral Operators. Stud. Math. 2016, 233, 279-291. [CrossRef]

8. Accomazzo, N.; Martínez-Perales, J.C.; Rivera-Ríos, I.P. On Bloom-Type Estimates for Iterated Commutators of Fractional Integrals. Indiana Univ. Math. J. 2020, 69, 1207-1230. [CrossRef]

9. Sato, S. Estimate for Singular Integrals on Homegeneous Groups. J. Math. Anal. Appl. 2013, 400, 311-330. [CrossRef]

10. Duong, X.T.; Li, H.Q.; Li, J.; Wick, B.D.; Wu, Q.Y. Lower Bound of Riesz Transform Kernels Revisited and Commutators on Stratified Lie Groups. arXiv 2018, arXiv:1803.01301.

11. Fan, Z.; Li, J. Quantitative Weighted Estimates for Rough Singular Integrals on Homogeneous Groups. arXiv 2020, arXiv:2009.02433.

12. Duong, X.T.; Gong, R.; Kuffner, M.S.; Li, J.; Wick, B.D.; Yang, D. Two Weight Commutators on Spaces of Homogeneous Type and Applications. J. Geom. Anal. 2021, 31, 980-1038. [CrossRef]

13. Coifman, R.R.; Weiss, G. Analyse Harmonique Non-Commutative Sur Certains Espaces Homogènes: Étude De Certaines Intégrales Singulières. In Lecture Notes in Mathematics; Springer: Heidelberg/Berlin, Germany, 1971; Volume 242.

14. Kairema, A.; Li, J.; Pereyra, M.C.; Ward, L.A. Haar Bases on Quasi-Metric Measure Spaces, and Dyadic Structure Theorems for Function Spaces on Product Spaces of Homogeneous Type. J. Funct. Anal. 2016, 271, 1793-1843. [CrossRef]

15. Cruz-Uribe, D.; Moen, K. One and two weighted norm inequalities for Riesz potentials. Ill. J. Math. 2013, 57, $295-323$.

16. Lerner, A.K.; Ombrosi, S.; Rivera-Ríos, I.P. Commutators of Singular Integrals Revisited. Bull. Lond. Math. Soc. 2019, 51, 107-119. [CrossRef]

17. Cruz-Uribe, D.; Martell, J.M.; Pérez, C. Sharp Weighted Estimates for Classical Operators. Adv. Math. 2012, $229,408-441$. [CrossRef] 